\title{
ORIGINAL
}

Lalitha Chattopadhyay

\section{Analytical solution for bending stress intensity factor in an orthotropic elastic plate containing a crack and subjected to concentrated moments}

Received: 5 April 2006 / Accepted: 6 September 2006 / Published online: 18 October 2006

(C) Springer-Verlag 2006

\begin{abstract}
The problem of estimating the bending stress distribution in the neighborhood of a crack located on a single line in an orthotropic elastic plate of constant thickness subjected to out-of-plane concentrated moments is examined. Using classical plate theory and integral transform techniques, the general formulae for the bending moment and twisting moment in an elastic plate containing cracks located on a single line are derived. The solution is obtained in a closed form for the case in which there is a single crack in an infinite plate subjected to symmetric concentrated moments.
\end{abstract}

Keywords Bending $\cdot$ Orthotropic plate $\cdot$ Crack $\cdot$ Integral transform $\cdot$ Stress intensity factor

\section{Introduction}

Fracture of plates and shells is of great practical as well as theoretical interest. The simplest approach to the out-of-plane fracture problems is to assume the small-deflection, Kirchhoff plate theory. The solution of the thin plate-bending problem was pioneered by Williams [1], who made use of the eigenfunction expansion technique and determined the stress distribution in the neighborhood of a crack. Using finite-element methods, Viz et al. [2], Alberto Zucchini et al. [3] computed stress intensity factors for thin cracked plates. Approximate weight functions are applied to investigate the influence of the orthotropy of the material on the fracture behavior of double cantilever beam in [4]. Using complex-variable methods Zehnder et al. [5] calculated stress intensity factor for a finite crack in an infinite isotropic plate. An analytical solution for the case of bending or twisting actions of an infinite orthotropic plate by moments that are uniformly distributed along the edges of the plate is given by the author in [6]. Using a similar analytical approach to that in the present study, the bending stress intensity factor is determined when the orthotropic plate (Fig. 1) is subjected to symmetric out-of-plane concentrated moments.

\section{Formulation of the problem}

Let us consider the bending action of an infinite plate by out-of-plane moments that are applied at an arbitrary location of the plate. Let the coordinate system be chosen so that the $x$-axis coincides with the line on which the cracks are located. The thickness of the plate is considered to be small in comparison with other dimensions. Let us assume that during bending, the plate undergoes a displacement $w$ perpendicular to the $x y$-plane. In the present analytical method, we consider the problem in which an infinite orthotropic elastic plate with the material principal axes aligned with respect to the coordinate axes. Let $M_{x x}, M_{x y}$ and $M_{y y}$ be the moment

L. Chattopadhyay $(\varangle)$

Structures Division, National Aerospace Laboratories, P.O. Box 1779, Kodhihalli, Bangalore 560 017, India

E-mail: lalitha@css.nal.res.in 


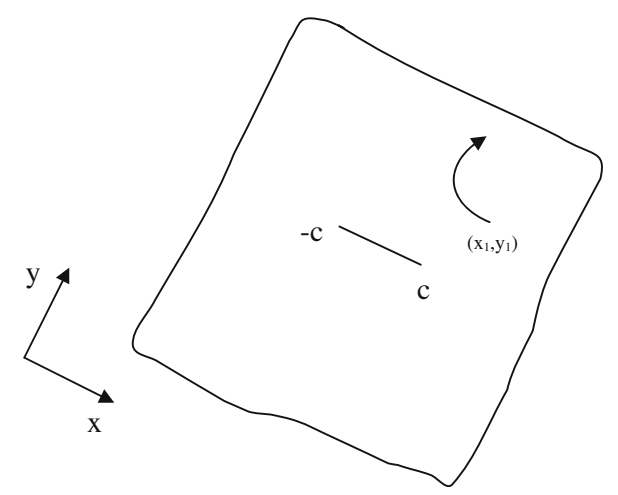

Fig. 1 Plate with a single crack subjected to concentrated moment at an arbitrary location $\left(x_{1}, y_{1}\right)$

resultants and $Q_{x}$ and $Q_{y}$ be the shear stress components. The crack surfaces are free from tractions and the stress in the plate is due to the action of applied moments.

\section{Analytical solution}

Let $L$ denote the union of intervals occupied by the cracks on the $x$-axis and $M$ be the interval not occupied by the cracks. The solution of the problem (Fig. 1) is obtained as the sum of the solutions of the following pair of problems. In the first problem (Fig. 2) the bending moment field satisfying the equations of equilibrium in the presence of applied concentrated moment tends to zero at infinity; it is assumed that there is no crack in the plate. The bending moment is determined in terms of the applied concentrated moment. In the second problem (Fig. 3), it is assumed that there is no concentrated moment applied in the plate and the crack surfaces are subjected to a self-equilibrating load system. In the second problem, we determine the bending moment in an infinite orthotropic plate containing cracks located on a single line. Fourier transform methods are employed to reduce the problem to that of a singular integral equation of Cauchy type. Let $f_{x}(x, y)$ and $f_{y}(x, y)$ be the applied out-of-plane moments about the $y$-axis and $x$-axis respectively, at an arbitrary point $(x, y)$ outside $L$. The crack surfaces are free from tractions:

$$
M_{y y}=0, \quad x \in L
$$

The moment resultants and displacement components vanish as $x^{2}+y^{2} \rightarrow \infty$.

In problem 1, we solve the equilibrium equations under the action of prescribed moments. Let $M_{x x}^{(1)}, M_{y y}^{(1)}, M_{x y}^{(1)}$ be the bending and twisting moments for this problem and hence on the crack line $y=0$, we have

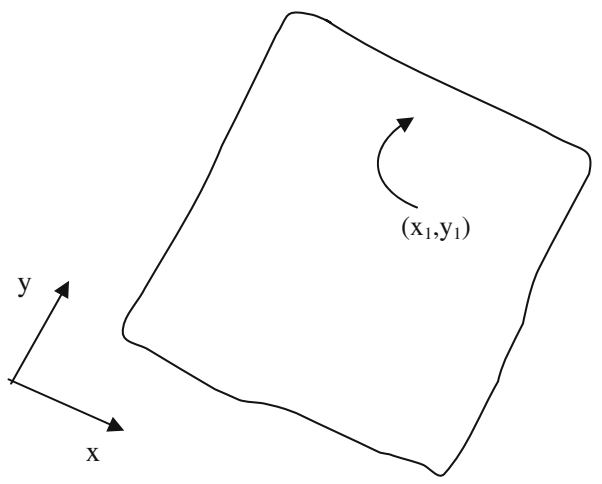

Fig. 2 Plate subjected to concentrated moment at an arbitrary location $\left(x_{1}, y_{1}\right)$ 


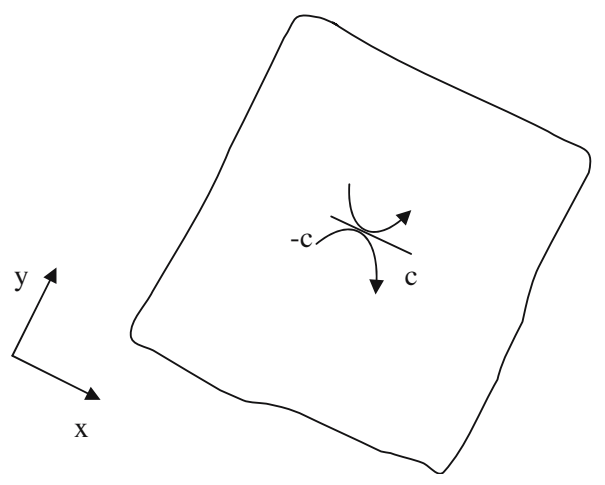

Fig. 3 Plate containing a single crack subjected to a self-equilibrating load system

$$
\begin{aligned}
& M_{y y}^{(1)}=-\frac{1}{2} H^{*}(x) \\
& M_{x y}^{(1)}=-\frac{1}{2} G^{*}(x)
\end{aligned}
$$

Where $G^{*}(x)$ and $H^{*}(x)$ are determined in terms of $f_{x}(x, y)$ and $f_{y}(x, y)$.

The moment-curvature relations in an orthotropic plate are given by

$$
\begin{aligned}
& M_{x x}=-z\left(C_{11} \frac{\partial^{2} w}{\partial x^{2}}+C_{12} \frac{\partial^{2} w}{\partial y^{2}}\right) \\
& M_{y y}=-z\left(C_{22} \frac{\partial^{2} w}{\partial y^{2}}+C_{12} \frac{\partial^{2} w}{\partial x^{2}}\right) \\
& M_{x y}=-2 z C_{66} \frac{\partial^{2} w}{\partial x \partial y}
\end{aligned}
$$

where $C_{11}, C_{12}, C_{22}, C_{66}$ are elastic constants of the material and are defined as follows:

$$
\begin{aligned}
& C_{11}=\frac{1}{E_{y} \Delta_{0}} ; \quad C_{22}=\frac{1}{E x \Delta_{0}} ; \quad C_{66}=G_{x y} \\
& C_{12}=\frac{v_{y x}}{E_{y} \Delta_{0}}=\frac{v_{x y}}{E_{x} \Delta_{0}} ; \quad \Delta_{0}=\frac{1-v_{y x} v_{x y}}{E_{x} E_{y}}
\end{aligned}
$$

where $E_{x}$ and $E_{y}$ are Young's moduli in the directions of the $x$ and $y$ axes, respectively. $G_{x y}$ is the shear modulus for a plane parallel to the $x y$-plane. $v_{x y}$ is the Poisson ratio characterizing the contraction in the direction of the $y$-axis when the tension is applied in the direction of the $x$-axis. Likewise, $v_{y x}$ is the Poisson ratio characterizing the contraction in the direction of the $x$-axis when the tension is applied in the direction of the $y$-axis.

In problem 2, we determine the bending and twisting moments $M_{x x}^{(2)}, M_{y y}^{(2)}, M_{x y}^{(2)}$ in the presence of cracks. It is assumed that there is no applied moment acting in the plate and the crack surfaces are subjected to a self-equilibrating load system

$$
\begin{aligned}
& M_{x y}^{(2)}=\frac{1}{2} G^{*}(x), \quad x \in L \\
& M_{y y}^{(2)}=\frac{1}{2} H^{*}(x), \quad x \in L
\end{aligned}
$$

If $Q_{x}$ and $Q_{y}$ are the shearing forces per unit length then the governing equations of bending effect are given by

$$
\begin{gathered}
\frac{\partial M_{x y}}{\partial x}-\frac{\partial M_{y y}}{\partial y}+Q_{y}=0 \\
\frac{\partial M_{y x}}{\partial y}+\frac{\partial M_{x x}}{\partial x}-Q_{x}=0 \\
\frac{\partial Q_{x}}{\partial x}+\frac{\partial Q_{y}}{\partial y}=0
\end{gathered}
$$


From (9) and (1), the Kirchhoff boundary condition given by the equation

$$
Q_{y}-\frac{\partial M_{y x}}{\partial x}=0, \quad \mathrm{y}=0, x \in L
$$

is also satisfied on free edges of the crack surfaces. The stress components at the top or bottom at $z=h / 2$ or $z=-h / 2$ are

$$
\sigma_{x y}=\frac{6 M_{x y}}{h^{2}} ; \quad \sigma_{y y}=\frac{6 M_{y y}}{h^{2}} ; \quad \sigma_{x x}=\frac{6 M_{x x}}{h^{2}}
$$

\section{Solution of problem 1}

The equations of equilibrium in the presence of out-of-plane concentrated moments is given by Jean-Marie Berthelot [7]

$$
\begin{aligned}
& \frac{\partial M_{x x}^{(1)}}{\partial x}+\frac{\partial M_{x y}^{(1)}}{\partial y}+f_{x}(x, y)=0 \\
& \frac{\partial M_{x y}^{(1)}}{\partial x}+\frac{\partial M_{y y}^{(1)}}{\partial y}+f_{y}(x, y)=0
\end{aligned}
$$

where $f_{x}, f_{y}$ are the applied moments at an arbitrary point $(x, y)$. by

Using Fourier transform techniques the twisting moment resultant [Appendix A, (A25) and (A26)] is given

$$
\begin{aligned}
& M_{x y}^{(1)}=\left(t_{2}^{2}-t_{1}^{2}\right)^{-1}\left[t_{1} T_{1}\left(x, y ; t_{1}, t_{2}\right)-t_{2} T_{1}\left(x, y ; t_{2}, t_{1}\right)\right] \\
& T_{1}(x, y ; p, q)= \frac{1}{2 \pi} \int_{-\infty}^{\infty} \int_{-\infty}^{\infty}\left[\left(p^{2}+\frac{C_{12}}{C_{22}}\right)(y-v) f_{x}(u, v)-\left(q^{2}+\frac{C_{12}}{C_{22}}\right)(x-u) f_{y}(u, v)\right] \\
& \times\left[(x-u)^{2}+p^{2}(y-v)^{2}\right]^{-1} \mathrm{~d} u \mathrm{~d} v
\end{aligned}
$$

Similarly the bending moment resultants are given by

$$
\begin{gathered}
M_{x x}^{(1)}=\left(t_{2}^{2}-t_{1}^{2}\right)^{-1}\left[t_{1} T_{2}\left(x, y ; t_{1}, t_{2}\right)-t_{2} T_{2}\left(x, y ; t_{2}, t_{1}\right)\right] \\
M_{y y}^{(1)}=-\left(t_{2}^{2}-t_{1}^{2}\right)^{-1}\left[\frac{1}{t_{1}} T_{2}\left(x, y ; t_{1}, t_{2}\right)-\frac{1}{t_{2}} T_{2}\left(x, y ; t_{2}, t_{1}\right)\right] \\
T_{2}(x, y ; p, q)=\frac{1}{2 \pi} \int_{-\infty}^{\infty} \int_{-\infty}^{\infty}\left[\left(p^{2}+\frac{C_{12}}{C_{22}}\right)(x-u) f_{x}(u, v)+p^{2}\left(q^{2}+\frac{C_{12}}{C_{22}}\right)(y-v) f_{y}(u, v)\right] \\
\times\left[(x-u)^{2}+p^{2}(y-v)^{2}\right]^{-1} \mathrm{~d} u \mathrm{~d} v \\
t_{1}, t_{2} \text { are the roots of } t^{4}-2 \Delta_{1} t^{2}+\Delta_{2}=0 ; \\
\Delta_{1}=\frac{C_{11} C_{22}-C_{12}^{2}-2 C_{12} C_{66}}{2 C_{22} C_{66}} ; \quad \Delta_{2}=\frac{C_{11}}{C_{22}}
\end{gathered}
$$

The bending and twisting moments on the $x$-axis are given by

$$
\begin{array}{r}
G^{*}(x)=-2 \operatorname{Im}\left[t_{1} \varphi_{1}(x)+t_{2} \psi_{1}(x)\right] \\
H^{*}(x)=-2 \operatorname{Re}\left[\varphi_{1}(x)+\psi_{1}(x)\right]
\end{array}
$$




$$
\begin{gathered}
\varphi_{1}(x)=\frac{1}{2} \int_{-\infty}^{\infty} \int_{-\infty}^{\infty} \frac{1}{\pi t_{1}} \frac{\left(t_{2}^{2}-t_{1}^{2}\right)^{-1}}{\left(w_{1}-x\right)}\left[m_{1} f_{x}(u, v)+\mathrm{i} t_{1} m_{2} f_{y}(u, v)\right] \mathrm{d} u \mathrm{~d} v \\
\psi_{1}(x)=-\frac{1}{2} \int_{-\infty}^{\infty} \int_{-\infty}^{\infty} \frac{1}{\pi t_{2}} \frac{\left(t_{2}^{2}-t_{1}^{2}\right)^{-1}}{\left(w_{2}-x\right)}\left[m_{2} f_{x}(u, v)+\mathrm{i} t_{2} m_{1} f_{y}(u, v)\right] \mathrm{d} u \mathrm{~d} v \\
m_{1}=t_{1}^{2}+\frac{C_{12}}{C_{22}} ; \quad m_{2}=t_{2}^{2}+\frac{C_{12}}{C_{22}} ; \quad w_{1}=u+\mathrm{i} t_{1} v ; \quad w_{2}=u+\mathrm{i} t_{2} v
\end{gathered}
$$

\section{Solution of problem 2}

The displacements in the $x$ and $y$ directions at any point are given by the following expressions:

$$
\begin{aligned}
& u_{x}=-z \frac{\partial w}{\partial x} \\
& u_{y}=-z \frac{\partial w}{\partial y}
\end{aligned}
$$

We define the displacement boundary conditions as given by

$$
A(x)=0, \quad x \in M ; \quad B(x)=0, x \in M
$$

where the displacement discontinuities are defined by the functions $A(x), B(x)$

$$
\begin{array}{ll}
A(x)=\frac{\partial}{\partial x}\left[u_{x}^{(1)}(x, 0)-u_{x}^{(2)}(x, 0)\right], & x \in L \\
B(x)=\frac{\partial}{\partial x}\left[u_{y}^{(1)}(x, 0)-u_{y}^{(2)}(x, 0)\right], & x \in L
\end{array}
$$

and the superscripts (1) and (2) denote the components in the upper half-plane $y>0$ and lower half-plane $y<0$ respectively. Since the cracks are subjected to a self-equilibrating load system, we have

$$
M_{x y}^{(1)}(x, 0)=M_{x y}^{(2)}(x, 0), M_{y y}^{(1)}(x, 0)=M_{y y}^{(2)}(x, 0), \quad x \in L
$$

The condition of continuity outside the crack on the $x$-axis may be written as

$$
M_{x y}^{(1)}(x, 0)=M_{x y}^{(2)}(x, 0) ; M_{y y}^{(1)}(x, 0)=M_{y y}^{(2)}(x, 0), \quad x \in M
$$

Combining (27) and (28) we can write

$$
\begin{aligned}
& M_{x y}^{(1)}(x, 0)-M_{x y}^{(2)}(x, 0)=0, \quad-\infty<x<\infty \\
& M_{y y}^{(1)}(x, 0)-M_{y y}^{(2)}(x, 0)=0, \quad-\infty<x<\infty
\end{aligned}
$$

The strain compatibility equation is given by

$$
\frac{\partial^{2} \varepsilon_{x x}}{\partial y^{2}}+\frac{\partial^{2} \varepsilon_{y y}}{\partial x^{2}}=2 \frac{\partial^{2} \gamma_{x y}}{\partial x \partial y}
$$

If we define the moment resultants in terms of Airy's function $\varphi(x, y)$ as given by

$$
M_{x x}=\frac{\partial^{2} \varphi}{\partial y^{2}} ; \quad M_{y y}=\frac{\partial^{2} \varphi}{\partial x^{2}} ; \quad M_{x y}=\frac{\partial^{2} \varphi}{\partial x \partial y}
$$


then the governing equations (9)-(11) are satisfied. Also from the compatibility equation (31) and from moment-curvature relations (4)-(6), the present problem reduces to that of solving the bi-harmonic equation in $\varphi(x, y)$ :

$$
\nabla^{4} \varphi=0 ; \quad \nabla^{4} \varphi=\frac{\partial^{4} \varphi}{\partial y^{4}}+2 \Delta_{1} \frac{\partial^{4} \varphi}{\partial x^{2} \partial y^{2}}+\Delta_{2} \frac{\partial^{4} \varphi}{\partial x^{4}}
$$

Using Fourier transform techniques, the bending moment resultant in the upper half-plane $y>0$, in terms of the transformed functions $\bar{A}(\xi)$ and $\bar{B}(\xi)$ of the displacement discontinuity functions $A(x), B(x)$ [Eq. (B30) of Appendix B] is given by

$$
\begin{aligned}
M_{y y}^{(1)}(x, y)= & \frac{\Delta_{0}}{4 \pi\left(t_{2}^{2}-t_{1}^{2}\right)} \int_{-\infty}^{\infty}\left\{\left[\bar{A}(\xi)-\frac{\mathrm{i} \bar{B} \operatorname{sgn}(\xi)}{t_{1}}\right] \mathrm{e}^{-t_{1}|\xi| y}\right. \\
& \left.-\left[\bar{A}(\xi)-\frac{\mathrm{i} \bar{B} \operatorname{sgn}(\xi)}{t_{2}}\right] \mathrm{e}^{-t_{2}|\xi| y}\right\} \mathrm{e}^{-\mathrm{i} \xi x} \mathrm{~d} \xi, \quad y>0
\end{aligned}
$$

where $\Delta_{0}=C_{11}-\frac{C_{12}^{2}}{C_{22}}$.

Similarly, we get the bending moment resultant in the lower half-plane $y<0$.

Evaluating the inner integral in terms of $A(s)$ and $B(s)$ we get the bending moment resultant as given by

$$
\begin{aligned}
M_{y y}(x, y)= & \frac{\Delta_{0}}{2 \pi\left(t_{2}^{2}-t_{1}^{2}\right)} \int_{-\infty}^{\infty} A(s) y\left\{\frac{t_{1}}{\left[(x-s)^{2}+t_{1}^{2} y^{2}\right]}-\frac{t_{2}}{\left[(x-s)^{2}+t_{2}^{2} y^{2}\right]}\right\} \mathrm{d} s \\
& -\int_{-\infty}^{\infty} \frac{B(s)(x-s)}{t_{1} t_{2}}\left\{\frac{t_{1}}{\left[(x-s)^{2}+t_{1}^{2} y^{2}\right]}-\frac{t_{2}}{\left[(x-s)^{2}+t_{2}^{2} y^{2}\right]}\right\} \mathrm{d} s, \quad y \neq 0
\end{aligned}
$$

Similarly the twisting moment resultant is given by

$$
\begin{aligned}
M_{x y}(x, y)= & \frac{\Delta_{0}}{2 \pi\left(t_{2}^{2}-t_{1}^{2}\right)} \int_{-\infty}^{\infty}[A(s)(x-s)+y B(s) y] \\
& \times\left\{\frac{t_{1}}{\left[(x-s)^{2}+t_{1}^{2} y^{2}\right]}-\frac{t_{2}}{\left[(x-s)^{2}+t_{2}^{2} y^{2}\right]}\right\} \mathrm{d} s, \quad y \neq 0
\end{aligned}
$$

Where $A(s)$ and $B(s)$ are the unknown functions to be determined.

The curvature terms are given by

$$
\begin{aligned}
\frac{\partial^{2} w}{\partial x^{2}}= & -\frac{1}{2 \pi\left(t_{2}^{2}-t_{1}^{2}\right)} \int_{-\infty}^{\infty} A(s) y\left\{\frac{m_{1} t_{1}}{\left[(x-s)^{2}+t_{1}^{2} y^{2}\right]}-\frac{m_{2} t_{2}}{\left[(x-s)^{2}+t_{2}^{2} y^{2}\right]}\right\} \mathrm{d} s \\
& -\int_{-\infty}^{\infty} \frac{B(s)(x-s)}{t_{1} t_{2}}\left\{\frac{m_{1} t_{2}}{\left[(x-s)^{2}+t_{1}^{2} y^{2}\right]}-\frac{m_{2} t_{1}}{\left[(x-s)^{2}+t_{2}^{2} y^{2}\right]}\right\} \mathrm{d} s,
\end{aligned}
$$

where

$$
\begin{aligned}
& m_{1}=t_{1}^{2}+\frac{C_{12}}{C_{22}} ; \quad m_{2}=t_{2}^{2}+\frac{C_{12}}{C_{22}} \\
& \quad \frac{\partial^{2} w}{\partial x \partial y}=\frac{1}{2 \pi\left(t_{2}^{2}-t_{1}^{2}\right)} \int_{-\infty}^{\infty}[A(s)(x-s)+y B(s)]\left\{\frac{t_{1} m_{2}}{\left[(x-s)^{2}+t_{1}^{2} y^{2}\right]}-\frac{t_{2} m_{1}}{\left[(x-s)^{2}+t_{2}^{2} y^{2}\right]}\right\} \mathrm{d} s
\end{aligned}
$$


The limiting values as $y \rightarrow 0+$ and $y \rightarrow 0-$ of the bending and twisting moments along the crack line are given by

$$
\begin{gathered}
M_{y y}(x, 0)=-\frac{\Delta_{0}}{2 \pi t_{1} t_{2}\left(t_{1}+t_{2}\right)} \int_{-\infty}^{\infty} \frac{B(s)}{x-s} \mathrm{~d} s \\
M_{x y}(x, 0)=-\frac{\Delta_{0}}{2 \pi\left(t_{1}+t_{2}\right)} \int_{-\infty}^{\infty} \frac{A(s)}{x-s} \mathrm{~d} s \\
M_{x x}(x, 0)=-\frac{\Delta_{0}}{2 \pi\left(t_{1}+t_{2}\right)} \int_{-\infty}^{\infty} \frac{B(s)}{x-s} \mathrm{~d} s
\end{gathered}
$$

From the boundary conditions (2)-(3) and (39), (40) we get the singular integral equations

$$
\begin{gathered}
\int_{-L}^{L} \frac{A(s)}{x-s} \mathrm{~d} s=\frac{-\pi\left(t_{1}+t_{2}\right)}{\Delta_{0}} G^{*}(x), \quad x \in L \\
\int_{-L}^{L} \frac{B(s)}{x-s} \mathrm{~d} s=\frac{-\pi t_{1} t_{2}\left(t_{1}+t_{2}\right)}{\Delta_{0}} H^{*}(x), \quad x \in L
\end{gathered}
$$

for the determination of $A(s)$ and $B(s)$, and $\Delta_{0}=C_{11}-\frac{C_{12}^{2}}{C_{22}}$.

\section{Single crack problem}

Solving for $A(x)$ and $B(x)$ in terms of $G^{*}(x), H^{*}(x)$ and substituting $A(s)$ and $B(s)$ in (39), (40) the bending and twisting moments on the crack line, $|x|>c$, are given by

$$
\begin{aligned}
& M_{y y}(x, 0)=\frac{\operatorname{sgn}(x)}{2 \pi \sqrt{\left(x^{2}-c^{2}\right)}} \int_{-c}^{c} \frac{H^{*}(t) \sqrt{\left(c^{2}-t^{2}\right)}}{t-x} \mathrm{~d} t \\
& M_{x y}(x, 0)=\frac{\operatorname{sgn}(x)}{2 \pi \sqrt{\left(x^{2}-c^{2}\right)}} \int_{-c}^{c} \frac{G^{*}(t) \sqrt{\left(c^{2}-t^{2}\right)}}{t-x} \mathrm{~d} t
\end{aligned}
$$

Substituting the values of $H^{*}(t)$ and $G^{*}(t)$ in the above equations the bending and twisting moments in problem 2 is given by

$$
\begin{gathered}
M_{y y}=\operatorname{Re}\left[\phi_{4}(x)+\varphi_{4}(x)\right], \quad|x|>c \\
M_{x y}=\operatorname{Im}\left[t_{1} \phi_{4}(x)+t_{2} \varphi_{4}(x)\right], \quad|x|>c \\
\phi_{4}(x)=\frac{1}{2} \int_{-\infty}^{\infty} \int_{-\infty}^{\infty} \frac{1}{t_{1}\left(t_{2}^{2}-t_{1}^{2}\right) \pi}\left[m_{1} f_{x}+\mathrm{i} t_{1} m_{2} f_{y}\right] g_{1}(x, w) \mathrm{d} u \mathrm{~d} v \\
\varphi_{4}(x)=\frac{1}{2} \int_{-\infty}^{\infty} \int_{-\infty}^{\infty} \frac{1}{t_{2}\left(t_{2}^{2}-t_{1}^{2}\right) \pi}\left[m_{2} f_{x}+\mathrm{i} t_{2} m_{1} f_{y}\right] g_{1}(x, w) \mathrm{d} u \mathrm{~d} v \\
g_{1}(x, w)=\frac{1}{x-w}-\frac{\operatorname{sgn}(x)}{\sqrt{x^{2}-c^{2}}}\left[1-\frac{\sqrt{w^{2}-c^{2}}}{x-w}\right],|x|>c ; \quad w=u+\mathrm{i} v
\end{gathered}
$$




\section{Concentrated moments}

We consider a particular case of concentrated moments $f_{y 0}$ and $-f_{y 0}$ about the $x$-axis (Fig. 4) acting at an arbitrary point $(0, y 1)$ and $(0,-y 1)$ represented by the Dirac delta function $\delta$ :

$$
f_{y}(x, y)=f_{y 0} \delta(x) \delta(y-y 0) ; \quad f_{y}(x, y)=-f_{y 0} \delta(x) \delta\left(y+y_{0}\right)
$$

Substituting the values of $f_{y}$ into (46) the bending moment along the crack line for the combination of these two forces is given by

$$
M_{y y}(x, 0)=-\frac{f_{y 0}|x|}{\pi\left(t_{2}^{2}-t_{1}^{2}\right) \sqrt{\left(x^{2}-c^{2}\right)}}\left[\frac{m_{2} \sqrt{c^{2}+t_{1}^{2} y_{0}^{2}}}{x^{2}+t_{1}^{2} y_{0}^{2}}-\frac{m_{1} \sqrt{c^{2}+t_{2}^{2} y_{0}^{2}}}{x^{2}+t_{2}^{2} y_{0}^{2}}\right], \quad|x|>c
$$

The mode I stress intensity factor at $z=h / 2$ is given by

$$
K_{\mathrm{I}}=\operatorname{Lt}_{x \rightarrow c}\left\{\sqrt{[2(x-c)]} \sigma_{y y}(x, 0)\right\}=\frac{3 f_{y 0} \sqrt{2 c}}{\pi\left(t_{2}^{2}-t_{1}^{2}\right) h^{2}}\left[\frac{m_{2}}{\sqrt{c^{2}+t_{1}^{2} y_{0}^{2}}}-\frac{m_{1}}{\sqrt{c^{2}+t_{2}^{2} y_{0}^{2}}}\right]
$$

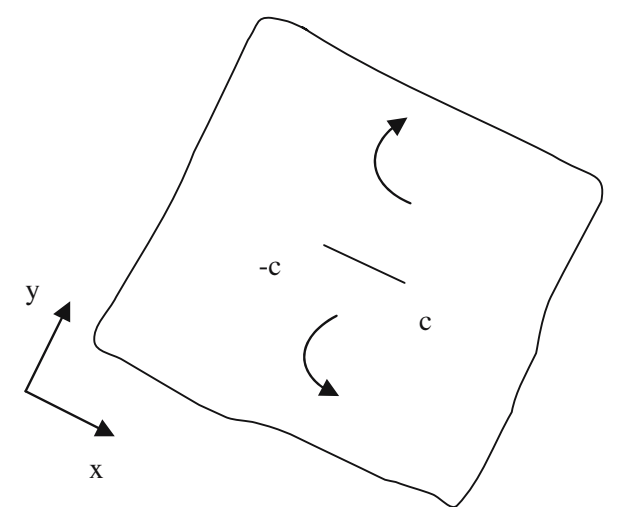

Fig. 4 Plate containing a crack and subjected to symmetric concentrated out-of-plane moments

\section{Uniformly distributed surface loading}

For isotropic material, let us consider the case of concentrated moment $f_{y 0}$ about the $x$-axis that acts at a point $\left(x_{1}, 0+\right)$ on the crack surface. By taking the limits as $y_{0} \rightarrow 0+,\left|x_{1}\right|<\mathrm{c}$ and from (49) for the upper half plate, we get

$$
K_{\mathrm{I}}=\frac{6 f_{y 0}}{2 \pi h^{2} \sqrt{c}} \frac{\sqrt{c+x_{1}}}{\sqrt{c-x_{1}}}
$$

If equal and opposite moments (Fig. 5) are added on to the lower half plate, thus forming a self-equilibrating load system, then the stress intensity factors are given by

$$
K_{\mathrm{I}}=\frac{6 f_{y 0}}{2 \pi h^{2} \sqrt{c}} \frac{\sqrt{c+x_{1}}}{\sqrt{c-x_{1}}}
$$

Letting $f_{y 0}=M_{y}(x, 0) \mathrm{d} x$ and integrating from $x=-c$ to $x=c$, the generalized expression for the opening mode (mode I) stress intensity factor is given by

$$
K_{\mathrm{I}}=\frac{6}{\pi h^{2} \sqrt{c}} \int_{-c}^{c} M_{y}(x, 0) \frac{\sqrt{c+x}}{\sqrt{c-x}} \mathrm{~d} x
$$




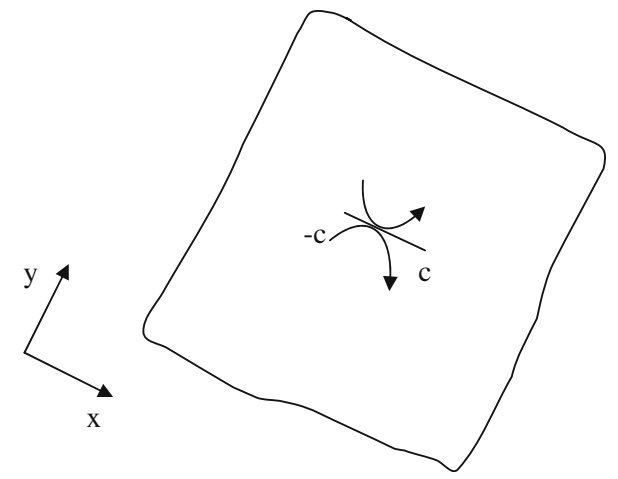

Fig. 5 Plate containing a crack and subjected to uniform out-of-plane moment on the crack surfaces

For the simple case of $M_{y}(x, 0)$ equal to uniform moment say, $M_{0}$, the mode I and mode II stress intensity factors are given by

$$
K_{\mathrm{I}}=\frac{6 M_{0} \sqrt{c}}{h^{2}}, \quad K_{\mathrm{II}}=0
$$

and the above solution is the same as that given in $[5,6,8]$.

\section{Conclusion}

A simple analytical approach for determining the analytical expression for the bending stress intensity factor in an orthotropic elastic plate containing a single crack and subjected to out-of-plane concentrated moments is explained. Using classical plate theory and integral transform techniques, the general formulae for the bending moment and twisting moment in an elastic plate containing cracks located on a single line are derived. The solution is obtained in detail for the case in which there is a single crack in an infinite plate and the bending stress intensity factor is determined in a closed form when the plate is subjected to concentrated out-of-plane moments.

\section{Appendix A}

$$
\begin{aligned}
& \frac{\partial M_{x x}^{(1)}}{\partial x}+\frac{\partial M_{x y}^{(1)}}{\partial y}+f_{x}(x, y)=0 \\
& \frac{\partial M_{x y}^{(1)}}{\partial x}+\frac{\partial M_{y y}^{(1)}}{\partial y}+f_{y}(x, y)=0
\end{aligned}
$$

The double Fourier transform of $f_{x}(x, y)$ is given by

$$
\bar{f}_{x}(\xi, \eta)=\int_{-\infty}^{\infty} \int_{-\infty}^{\infty} f_{x}(x, y) \mathrm{e}^{\mathrm{i} \xi x} \mathrm{e}^{\mathrm{i} \eta y} \mathrm{~d} x \mathrm{~d} y
$$

Taking the double Fourier transform of (A1) and (A2), we get

$$
\begin{aligned}
& \mathrm{i} \xi \bar{M}_{x x}^{(1)}+\mathrm{i} \eta \bar{M}_{x y}^{(1)}+\overline{f_{x}}(\xi, \eta)=0 \\
& \mathrm{i} \xi \bar{M}_{x y}^{(1)}+\mathrm{i} \eta \bar{M}_{y y}^{(1)}+\overline{f_{y}}(\xi, \eta)=0
\end{aligned}
$$


The displacement components are given by

$$
\begin{gathered}
u_{x}=-z \frac{\partial w}{\partial x} \\
u_{y}=-z \frac{\partial w}{\partial y} \\
\bar{M}_{x x}=\mathrm{i}\left(C_{11} \xi \bar{u}_{x}+C_{12} \eta \bar{u}_{y}\right) \\
\bar{M}_{y y}=\mathrm{i}\left(C_{12} \xi \bar{u}_{x}+C_{22} \eta \bar{u}_{y}\right) \\
\bar{M}_{x y}=\mathrm{i} C_{66}\left(\bar{u}_{y} \xi+\bar{u}_{x} \eta\right) \\
\bar{u}_{x}=C(\xi, \eta)\left\{\bar{f}_{x}\left(C_{66} \xi^{2}+C_{22} \eta^{2}\right)-\bar{f}_{y} \xi \eta\left(C_{66}+C_{12}\right)\right\} \\
\bar{u}_{y}=C(\xi, \eta)\left\{\bar{f}_{y}\left(C_{66} \eta^{2}+C_{11} \xi^{2}\right)-\bar{f}_{x} \xi \eta\left(C_{66}+C_{12}\right)\right\}
\end{gathered}
$$

where

$$
C(\xi, \eta)=\left[C_{22} C_{66}\left(\xi^{2} t_{1}^{2}+\eta^{2}\right)\left(\xi^{2} t_{2}^{2}+\eta^{2}\right)\right]^{-1}
$$

Using the above equations, the double Fourier transform of the bending moment and twisting moments (A9)-(A10) become

$$
\begin{aligned}
\bar{M}_{x x}= & -\mathrm{i} C(\xi, \eta)\left\{\xi \overline{f_{x}}(\xi, \eta)\left[C_{11} C_{66} \xi^{2}+\eta^{2}\left(C_{11} C_{22}-C_{12} C_{66}-C_{12}^{2}\right)\right]+\eta \overline{f_{y}} C_{66}\left[C_{12} \eta^{2}-C_{11} \xi^{2}\right]\right\} \\
\bar{M}_{y y}= & -\mathrm{i} C(\xi, \eta)\left\{\xi \overline{f_{x}}(\xi, \eta) C_{66}\left[C_{12} \xi^{2}-C_{22} \eta^{2}\right]+\eta \overline{f_{y}}\left[C_{22} C_{66} \eta^{2}+\xi^{2}\left(C_{11} C_{22}-C_{12} C_{66}-C_{12}^{2}\right)\right]\right\} \\
& \bar{M}_{x y}=\mathrm{i} C(\xi, \eta) C_{66}\left\{\xi \overline{f_{x}}(\xi, \eta)\left[\eta \overline{f_{x}} C_{66} C_{12} \xi^{2}-C_{22} \eta^{2}\right]+\xi \overline{f_{y}} C_{66}\left[C_{12} \eta^{2}-C_{11} \xi^{2}\right]\right\}
\end{aligned}
$$

Taking the inverse transform of (A16), we get the twisting moment resultant as given by the following equation:

$$
\begin{aligned}
M_{x y}= & -\frac{i}{4 \pi^{2} C_{22}\left(t_{2}^{2}-t_{1}^{2}\right)} \int_{-\infty}^{\infty} \int_{-\infty}^{\infty}\left[f_{x}(u, v) I_{1}+f_{x}(u, v) I_{2}\right] \mathrm{d} u \mathrm{~d} v \\
I_{1}= & \int_{-\infty}^{\infty} \int_{-\infty}^{\infty}\left[\frac{\eta}{\xi^{2}}\left(C_{12} \xi^{2}-C_{22} \eta^{2}\right)\left\{\frac{1}{\xi^{2} t_{1}^{2}+\eta^{2}}-\frac{1}{\xi^{2} t_{1}^{2}+\eta^{2}}\right\}\right] \mathrm{e}^{-\mathrm{i} \xi(x-u)-\mathrm{i} \eta(y-v)} \mathrm{d} \xi \mathrm{d} \eta \\
I_{2}= & \int_{-\infty}^{\infty} \int_{-\infty}^{\infty}\left[\frac{1}{\xi}\left(C_{12} \eta^{2}-C_{11} \xi^{2}\right)\left\{\frac{1}{\xi^{2} t_{1}^{2}+\eta^{2}}-\frac{1}{\xi^{2} t_{1}^{2}+\eta^{2}}\right\}\right] \mathrm{e}^{-\mathrm{i} \xi(x-u)-\mathrm{i} \eta(y-v) \mathrm{d} \xi \mathrm{d} \eta} \\
I_{11}= & \int_{-\infty}^{\infty} \int_{-\infty}^{\infty} \frac{\eta}{\xi^{2} t^{2}+\eta^{2}} \mathrm{e}^{-\mathrm{i} \xi(x-u)-\mathrm{i} \eta(y-v)} \mathrm{d} \xi \mathrm{d} \eta=-4 \mathrm{i} \int_{0}^{\infty} \frac{\eta \cos \xi(x-u) \sin \eta(y-v)}{\xi_{0}^{\infty}} \mathrm{d} \xi \mathrm{d} \eta \\
I_{12}= & \int_{-\infty}^{\infty} \int_{-\infty}^{\infty} \frac{\eta^{3} t^{2}+\eta^{2}}{\xi^{2}\left(\xi^{2} t^{2}+\eta^{2}\right)} \mathrm{e}^{-\mathrm{i} \xi(x-u)-\mathrm{i} \eta(y-v)} \mathrm{d} \xi \mathrm{d} \eta=-4 \mathrm{i} \int_{0}^{\infty} \frac{\eta^{3} \cos \xi(x-u) \sin \eta(y-v)}{\xi^{2}\left(\xi^{2} t^{2}+\eta^{2}\right)} \mathrm{d} \xi \mathrm{d} \eta
\end{aligned}
$$

Using the integral

$$
\int_{0}^{\infty} \frac{\eta \sin (\eta y)}{\xi^{2} p^{2}+\eta^{2}} \mathrm{~d} \eta=\frac{\pi}{2} \mathrm{e}^{-p|\xi| y}
$$


we can evaluate the integral (A20) with respect to $\eta$ and (A20) reduces to

$$
\begin{aligned}
& I_{11}=-2 \pi \mathrm{i} \operatorname{sgn}(y-v) \int_{0}^{\infty} \mathrm{e}^{-\xi t|y-v|} \cos \xi(x-u) \mathrm{d} \xi=2 \pi \mathrm{i} \frac{t(y-v)}{(x-u)^{2}+t^{2}(y-v)^{2}} \\
& I_{12}=2 \pi \mathrm{i} \frac{t^{3}(y-v)}{(x-u)^{2}+t^{2}(y-v)^{2}}
\end{aligned}
$$

Using Eqs. $\mathrm{A}(18)-\mathrm{A}(21)$, we get the twisting moment resultant as given by

$$
M_{x y}^{(1)}=\left(t_{2}^{2}-t_{1}^{2}\right)^{-1}\left[t_{1} T_{1}\left(x, y ; t_{1}, t_{2}\right)-t_{2} T_{1}\left(x, y ; t_{2}, t_{1}\right)\right]
$$

where

$$
\begin{aligned}
T_{1}(x, y ; p, q)= & \frac{1}{2 \pi} \int_{-\infty}^{\infty} \int_{-\infty}^{\infty}\left[\left(p^{2}+\frac{C_{12}}{C_{22}}\right)(y-v) f_{x}(u, v)-\left(q^{2}+\frac{C_{12}}{C_{22}}\right)(x-u) f_{y}(u, v)\right] \\
& \times\left[(x-u)^{2}+p^{2}(y-v)^{2}\right]^{-1} \mathrm{~d} u \mathrm{~d} v
\end{aligned}
$$

\section{Appendix B}

The governing differential equation of problem (2) is given by the bi-harmonic equation in $\varphi(x, y)$ :

$$
\nabla^{4} \varphi=0 ; \quad \nabla^{4} \varphi=\frac{\partial^{4} \varphi}{\partial y^{4}}+2 \Delta_{1} \frac{\partial^{4} \varphi}{\partial x^{2} \partial y^{2}}+\Delta_{2} \frac{\partial^{4} \varphi}{\partial x^{4}}
$$

Let $\varphi^{(1)}(x, y)$ denote $\varphi(x, y)$ in the upper half-plane $y>0$ and $G^{(1)}(\xi, y)$ be the Fourier transform of $\varphi^{(1)}(x, y)$ and let $\varphi^{(2)}(x, y)$ denote $\varphi(x, y)$ in the lower half-plane $y<0$ and $G^{(2)}(\xi, y)$ be the Fourier transform of $\varphi^{(2)}(x, y)$.

$$
\begin{aligned}
& G^{(1)}(\xi, y)=\int_{-\infty}^{\infty} \varphi^{(1)}(x, y) \mathrm{e}^{\mathrm{i} \xi x} \mathrm{~d} x, \quad y>0 \\
& G^{(2)}(\xi, y)=\int_{-\infty}^{\infty} \varphi^{(2)}(x, y) \mathrm{e}^{\mathrm{i} \xi x} \mathrm{~d} x, \quad y<0
\end{aligned}
$$

Taking Fourier transformation of the bi-harmonic equation, we get the ordinary differential equation in $G(\xi, y)$ as given by

$$
\frac{\mathrm{d}^{4} G(\xi, y)}{\mathrm{d} y^{4}}-2 \Delta_{1} \xi^{2} \frac{\mathrm{d}^{2} G(\xi, y)}{\mathrm{d} y^{2}}+\Delta_{2} \xi^{4} G(\xi, y)=0
$$

The solutions for the above differential equation are given by the following expressions:

$$
\begin{aligned}
& G^{(1)}(\xi, y)=P_{1}(\xi) \mathrm{e}^{-t_{1}|\xi| y}+y Q_{1}(\xi) \mathrm{e}^{-t_{2}|\xi| y}, \quad y>0 \\
& G^{(2)}(\xi, y)=P_{2}(\xi) \mathrm{e}^{t_{1}|\xi| y}+y Q_{2}(\xi) \mathrm{e}^{t_{2}|\xi| y}, \quad y<0
\end{aligned}
$$

where the superscripts (1) and (2) indicate the upper and lower half-planes, respectively. The constants $t_{1}$ and $t_{2}$ are the real parts of the roots of the quartic equation

$$
t^{4}-2 \Delta_{1} t^{2}+\Delta_{2}=0
$$


$P_{1}(\xi), P_{2}(\xi), Q_{1}(\xi), Q_{2}(\xi)$ are the unknown functions to be determined from the boundary conditions and continuity conditions. The bending and twisting moments in terms of $G^{(1)}(\xi, y)$ for $y>0$ are given by

$$
\begin{aligned}
M_{x x}^{(1)}(x, y)=\frac{1}{2 \pi} \int_{-\infty}^{\infty} \frac{\partial^{2} G^{(1)}(\xi, y)}{\partial y^{2}} \mathrm{e}^{-\mathrm{i} \xi x} \mathrm{~d} \xi, & y>0 \\
M_{y y}^{(1)}(x, y)=\frac{1}{2 \pi} \int_{-\infty}^{\infty} \xi^{2} G^{(1)}(\xi, y) \mathrm{e}^{-\mathrm{i} \xi x} \mathrm{~d} \xi, & y>0 \\
M_{x y}^{(1)}(x, y)=-\frac{\mathrm{i}}{2 \pi} \int_{-\infty}^{\infty} \xi \frac{\partial G^{(1)}(\xi, y)}{\partial y} \mathrm{e}^{-\mathrm{i} \xi x} \mathrm{~d} \xi, & y>0
\end{aligned}
$$

The bending and twisting moments for $y<0$ in terms of $G^{(2)}(\xi, y)$ are given by

$$
\begin{aligned}
& M_{x x}^{(2)}(x, y)=\frac{1}{2 \pi} \int_{-\infty}^{\infty} \frac{\partial^{2} G^{(2)}(\xi, y)}{\partial y^{2}} \mathrm{e}^{-\mathrm{i} \xi x} \mathrm{~d} \xi, \quad y<0 \\
& M_{y y}^{(2)}(x, y)=\frac{1}{2 \pi} \int_{-\infty}^{\infty} \xi^{2} G^{(2)}(\xi, y) \mathrm{e}^{-\mathrm{i} \xi x} \mathrm{~d} \xi, \quad y<0 \\
& M_{x y}^{(2)}(x, y)=-\frac{\mathrm{i}}{2 \pi} \int_{-\infty}^{\infty} \xi \frac{\partial G^{(2)}(\xi, y)}{\partial y} \mathrm{e}^{-\mathrm{i} \xi x} \mathrm{~d} \xi, \quad y<0
\end{aligned}
$$

The partial derivatives with respect to $x$ of the displacement components in the upper half-plane $(y>0)$ are given by

$$
\begin{aligned}
& \frac{\partial}{\partial x} u_{x}^{(1)}(x, y)=\frac{1}{2 \pi \Delta_{0}} \int_{-\infty}^{\infty}\left[\frac{\partial^{2}}{\partial y^{2}} G^{(1)}+\frac{C_{12}}{C_{22}} \xi^{2} G^{(1)}\right] \mathrm{e}^{-\mathrm{i} \xi x} \mathrm{~d} \xi \\
& \frac{\partial}{\partial x} u_{y}^{(1)}(x, y)=-\frac{\mathrm{i}}{2 \pi \Delta_{0}} \int_{-\infty}^{\infty}\left[\frac{\partial^{3}}{\partial y^{3}} G^{(1)}+\left(\frac{C_{12}}{C_{22}}-\frac{\Delta_{0}}{C_{66}}\right) \frac{\partial}{\partial y} G^{(1)}\right] \mathrm{e}^{-\mathrm{i} \xi x} \mathrm{~d} \xi
\end{aligned}
$$

The partial derivatives with respect to $x$ of the displacement components in the lower half-plane $(y<0)$ is given by

$$
\begin{aligned}
& \frac{\partial}{\partial x} u_{x}^{(2)}(x, y)=\frac{1}{2 \pi \Delta_{0}} \int_{-\infty}^{\infty}\left[\frac{\partial^{2}}{\partial y^{2}} G^{(2)}+\frac{C_{12}}{C_{22}} \xi^{2} G^{(2)}\right] \mathrm{e}^{-\mathrm{i} \xi x} \mathrm{~d} \xi \\
& \frac{\partial}{\partial x} u_{y}^{(2)}(x, y)=-\frac{\mathrm{i}}{2 \pi \Delta_{0}} \int_{-\infty}^{\infty}\left[\frac{\partial^{3}}{\partial y^{3}} G^{(2)}+\left(\frac{C_{12}}{C_{22}}-\frac{\Delta_{0}}{C_{66}}\right) \frac{\partial}{\partial y} G^{(2)}\right] \mathrm{e}^{-\mathrm{i} \xi x} \mathrm{~d} \xi
\end{aligned}
$$

The displacement discontinuities are given by the equations

$$
\begin{array}{ll}
A(x)=\frac{\partial}{\partial x}\left[u_{x}^{(1)}(x, 0)-u_{x}^{(2)}(x, 0)\right], & x \in L \\
B(x)=\frac{\partial}{\partial x}\left[u_{y}^{(1)}(x, 0)-u_{y}^{(2)}(x, 0)\right], & x \in L
\end{array}
$$


The moment boundary conditions are given by

$$
\begin{aligned}
& M_{x y}^{(1)}(x, 0)-M_{x y}^{(2)}(x, 0)=0, \quad-\infty<x<\infty \\
& M_{y y}^{(1)}(x, 0)-M_{y y}^{(2)}(x, 0)=0, \quad-\infty<x<\infty
\end{aligned}
$$

Using the displacement discontinuity relations (B18)-(B19), the moment boundary conditions (B20)-(B21) and using (B8)-(B13), we get the algebraic equations for solving the four unknowns $P_{1}(\xi), P_{2}(\xi), Q_{1}(\xi), Q_{2}(\xi)$ appearing in $G^{(1)}$ and $G^{(2)}$ in terms of $\bar{A}(\xi)$ and $\bar{B}(\xi)$ as given by the following equations:

$$
\begin{gathered}
P_{1}(\xi)+Q_{1}(\xi)=P_{2}(\xi)+Q_{2}(\xi) \\
t_{1} P_{1}(\xi)+t_{2} Q_{1}(\xi)+t_{1} P_{2}(\xi)+t_{2} Q_{2}(\xi)=0 \\
\Delta_{0} \bar{A}(\xi)=\xi^{2}\left[t_{1}^{2} P_{1}(\xi)+t_{2}^{2} Q_{1}(\xi)-t_{1}^{2} P_{2}(\xi)-t_{2}^{2} Q_{2}(\xi)\right]+\frac{C_{12}}{C_{22}} \xi^{2}\left[P_{1}(\xi)+Q_{1}(\xi)-P_{2}(\xi)-Q_{2}(\xi)\right] \\
\Delta_{0} \bar{B}(\xi)=\mathrm{i} \xi^{2} \operatorname{sgn}(\xi)\left[t_{1}^{3} P_{1}(\xi)+t_{2}^{3} Q_{1}(\xi)+t_{1}^{3} P_{2}(\xi)+t_{2}^{3} Q_{2}(\xi)\right] \\
+\left[\frac{C_{12}}{C_{22}}-\frac{\Delta_{0}}{C_{66}}\right]\left[t_{1} P_{1}(\xi)+t_{2} Q_{1}(\xi)+t_{1} P_{2}(\xi)+t_{2} Q_{2}(\xi)\right]
\end{gathered}
$$

Solving the above equations we get

$$
\begin{aligned}
P_{1}(\xi) & =-\frac{\Delta_{0}}{2\left(t_{2}^{2}-t_{1}^{2}\right) \xi^{2}}\left[\bar{A}(\xi)-\frac{\mathrm{i}}{t_{1}} \bar{B}(\xi) \operatorname{sgn}(\xi)\right] \\
Q_{1}(\xi) & =\frac{\Delta_{0}}{2\left(t_{2}^{2}-t_{1}^{2}\right) \xi^{2}}\left[\bar{A}(\xi)-\frac{\mathrm{i}}{t_{2}} \bar{B}(\xi) \operatorname{sgn}(\xi)\right] \\
P_{2}(\xi) & =\frac{\Delta_{0}}{2\left(t_{2}^{2}-t_{1}^{2}\right) \xi^{2}}\left[\bar{A}(\xi)+\frac{\mathrm{i}}{t_{1}} \bar{B}(\xi) \operatorname{sgn}(\xi)\right] \\
Q_{2}(\xi) & =-\frac{\Delta_{0}}{2\left(t_{2}^{2}-t_{1}^{2}\right) \xi^{2}}\left[\bar{A}(\xi)+\frac{\mathrm{i}}{t_{2}} \bar{B}(\xi) \operatorname{sgn}(\xi)\right]
\end{aligned}
$$

Substituting the above values of $P_{1}(\xi)$ and $Q_{1}(\xi)$ in (B5) and (B9), we get the bending moment resultant for $y>0$, as given by

$M_{y y}^{(1)}(x, y)=\frac{\Delta_{0}}{4 \pi\left(t_{2}^{2}-t_{1}^{2}\right)} \int_{-\infty}^{\infty}\left\{\left[\bar{A}(\xi)-\frac{\mathrm{i} \bar{B} \operatorname{sgn}(\xi)}{t_{1}}\right] \mathrm{e}^{-t_{1}|\xi| y}-\left[\bar{A}(\xi)-\frac{\mathrm{i} \bar{B} \operatorname{sgn}(\xi)}{t_{2}}\right] \mathrm{e}^{-t_{2}|\xi| y}\right\} \mathrm{e}^{-\mathrm{i} \xi x} \mathrm{~d} \xi, y>0$

Similarly substituting the above values of $P_{2}(\xi)$ and $Q_{2}(\xi)$ in (B6) and (B12), we get the bending moment resultant for $y<0$, as given by

$$
M_{y y}^{(2)}(x, y)=\frac{-\Delta_{0}}{4 \pi\left(t_{2}^{2}-t_{1}^{2}\right)} \int_{-\infty}^{\infty}\left\{\left[\bar{A}(\xi)+\frac{\mathrm{i} \bar{B} \operatorname{sgn}(\xi)}{t_{1}}\right] \mathrm{e}^{t_{1}|\xi| y}-\left[\bar{A}(\xi)+\frac{i \bar{B} \operatorname{sgn}(\xi)}{t_{2}}\right] \mathrm{e}^{t_{2}|\xi| y}\right\} \mathrm{e}^{-\mathrm{i} \xi x} \mathrm{~d} \xi, y<0
$$




\section{References}

1. Williams, M.L.: The bending stress distribution at the base of a stationary crack. ASME J. Appl. Mech. 28, 78-82 (1961)

2. Viz, M.J., Potyondy, D.O., Zehnder, A.T, Rankin, C.C., Riks, E.: Int. J. Fract. 72, 21-38 (1995)

3. Zucchini, A., Hui, C.Y., Zehnder, A.T.: Crack tip stress fields for thin plates in bending, shear and twisting. A three dimensional finite element study. Int. J. Fract. 104, 387-407 (2000)

4. Massabò, R., Brandinelli, L., Cox, B.N.: Mode I weight functions for an orthotropic double cantilever beam. Int. J. Eng. Sci. 41, 1497-1518 (2003)

5. Zehnder, A.T., Hui, C.Y.: Stress intensity factors for plate bending and shearing problems. J. Appl. Mech. 61, 719-722 (1994)

6. Lalitha Chattopadhyay: Analytical solution for an orthotropic elastic plate containing cracks. Int. J. Fract. 134, 305-317 (2005)

7. Berthelot, J.-M.: Composite Materials. Springer, Berlin Heidelberg New York (1998)

8. Murakami, Y.: Stress Intensity Factors Handbook, 2. Pergamon, Oxford (1987) 